\title{
THE ADDED MASS COEFFICIENT OF A DISPERSION OF SPHERICAL GAS BUBBLES IN LIQUID
}

\author{
A. Biesheuvel ${ }^{1}$ and S. Spoelstra ${ }^{2}$ \\ Departments of 'Mechanical Engineering and 'Applied Physics, University of Twente, P.O. Box 217, \\ 7500 AE Enschede. The Netherlands
}

(Received 5 January 1988; in revised form 26 February 1989)

\begin{abstract}
Models published in the two-phase flow literature for the added mass coefficient of a dilute bubbly dispersion are discussed and compared. It is shown that the differences between the models are mainly due to the different ways in which the added mass is defined. Also, approximate expressions for the added mass coefficient of non-dilute bubbly dispersions are given. Finally, the use of the models in an equation for the average motion of the bubbles is briefly discussed.
\end{abstract}

Key Words: bubbly flows, added mass

\section{INTRODUCTION}

Conservation equations for the flow of dispersions of gas bubbles in liquid include an added mass coefficient, when forces associated with the inertia of the fluid are of importance to the bubble motion. Due to hydrodynamic interactions between the gas bubbles this coefficient depends on the volumetric gas concentration or void fraction, and various relations expressing this dependency have been proposed, particularly for dilute dispersions. It is the purpose of this paper, firstly, to show that the differences between these relations are essentially due to differences in the definition of an effective added mass for a bubbly dispersion and the approximations made in their derivation. For a single rigid body in translational motion in a steady unbounded flow field of an effectively inviscid fluid, the concept of added mass may be defined through that of the impulse to be transmitted to the fluid by the action on the body over a very short time of some hypothetical very large force, in order to set up the flow field instantaneously from a situation in which the body is imagined to move with the fluid velocity at infinity, i.e. one in which the body experiences no force. Alternatively, one may study how the presence of the fluid affects the velocity that the body will acquire upon an instantaneous acceleration of the fluid at infinity. For a single rigid body the two methods are equivalent, in contrast to the case of a bubbly dispersion, as will be shown. Illuminating discussions of the added mass concept are given in Lighthill (1986) and Landau \& Lifshitz (1959); the exposition in this paper owes much to these books.

A well-known expression for the added mass coefficient $m(\epsilon)$ of a dilute bubbly dispersion consisting of equally-sized spheres is (van Wijngaarden 1976)

$$
m(\epsilon)=\frac{1}{2} \rho_{1} \epsilon(1+2.78 \epsilon)+O\left(\epsilon^{3}\right) .
$$

Here $\rho_{1}$ and $\epsilon$ denote the density of the fluid and the void fraction, respectively. van Wijngaarden (1976) determined the mean velocity of the bubbles of a uniform dispersion immediately after the flow had been impulsively set into motion. By calculating the velocity that a reference bubbles will attain in the presence of just one other bubble at an arbitrary relative position, and by subsequently averaging the result over all possible relative positions of the two bubbles, [1] was obtained. The method will be briefly explained in section 3.1 for the more general case in which the dispersion is not necessarily at rest initially.

The alternative way of defining the effective added mass, through the fluid impulse, is treated in section 3.2, again for a dilute dispersion. In contrast to the one used by van Wijngaarden, for which only the probability distribution of the relative bubbles positions has to be specified, this method also requires a specification of the probability distribution of the relative bubble velocities. We have not been able to find any information on this distribution function, neither for dilute nor 
for non-dilute dispersions, and shall here make the usual step of assuming the bubbles to have equal velocities. The resulting fluid dynamical problem is then analogous to that encountered in the determination of the effective thermal conductivity of a composite material consisting of equallysized non-conducting spheres in a conducting matrix. For a dilute composite the latter problem was addressed by Jeffrey (1973); by a minor adjustment of his analysis the added mass coefficient is found to be given by

$$
m(\epsilon)=\frac{1}{2} \rho_{1} \epsilon(1+3.32 \epsilon)+O\left(\epsilon^{3}\right) .
$$

As will be explained, the difference between [1] and [2] is due to the fact that different velocity probability distributions are used in their derivation. van Wijngaarden's (1976) result is exact to the extent that the bubble velocities are defined by the dynamics of the flow problem, whereas for the determination of the fluid impulse in section 3.2 an assumption for the velocity distribution function has been made. When the precise details of the bubble interactions are not included, but merely the presence of neighbouring bubbles around a reference bubble is accounted for, Zuber's (1964) result is found,

$$
m(c)=\frac{1}{2} \rho_{1} \epsilon\left(\frac{1+2 \epsilon}{1-\epsilon}\right)
$$

which in the limit of small void fraction reduces to

$$
m(\epsilon)=\frac{1}{2} \rho_{1} \epsilon(1+3 \epsilon)+O\left(\epsilon^{3}\right) .
$$

The second purpose of this paper is to present an expression for the added mass coefficient of a non-dilute bubbly dispersion. Both the forementioned approaches lead to the same result, valid for any value of the void fraction, when the spherical bubbles are taken to be arranged in cubic arrays of infinite extent. By symmetry, all bubbles have equal velocities in that case. Exact relations for the added mass coefficient for three types of cubic arrays will be given in section 4.1 through an analysis based on Sangani \& Acrivos $(1982,1983)$. An interpolation then yields (section 4.2) an approximate relation for the added mass coefficient for random non-dilute dispersions.

We conclude the paper (section 5) with a brief discussion on the use of the relations that were under consideration in an equation of motion for the gas bubbles.

The description of the flow of a bubbly dispersion in this paper is not in terms of the drift flux model or the multifield model. Flow parameters will be defined as ensemble averages, and instead of the mean fluid velocity we will take the mean velocity of the dispersion as a reference velocity for the bubble motion. In order to avoid misunderstanding a brief comment on this method of description is given in the following section (section 2).

\section{THE METHOD OF AVERAGING}

Equations of motion for bubbly dispersions that treat the two phases as continua are necessarily relations between rates of change of averaged flow parameters. This implies that some kind of averaging procedure is involved in their derivation. The appropriate method of averaging for dispersed flows, a bubbly fluid for example, is statistical averaging over an ensemble of realizations of the dispersion (cf. Batchelor 1970). The usual procedure is then to introduce a probability density function (pdf) $f\left(\mathscr{B}_{N}, \mathscr{V}_{N}, t\right)$ meaning that

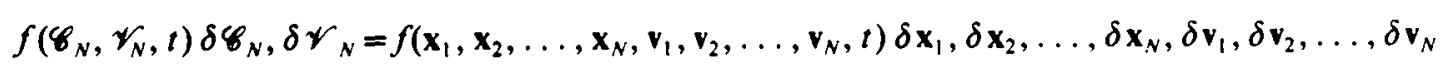

is the probability of finding $N$ bubbles, where $N \gg 1$, at time $t$ in the volume elements $\delta \mathbf{x}_{1}$, $\delta \mathbf{x}_{2}, \ldots, \delta \mathbf{x}_{N}, \delta \mathbf{v}_{1}, \delta \mathbf{v}_{2}, \ldots, \delta \mathbf{v}_{N}$ around the points $\mathbf{x}_{1}, \mathbf{x}_{2}, \ldots, \mathbf{x}_{N}, \mathbf{v}_{1}, \mathbf{v}_{2}, \ldots, \mathbf{v}_{N}$ of a $6 N-$ dimensional phase space. Each point in this space specifies the positions of the centres and the velocities of the $N$ bubbles in one realization of the ensemble. In this paper the bubbles will be assumed to be identical rigid spheres, so the pdf is normalized by

$$
\iint f\left(\mathscr{B}_{N}, \mathscr{V}_{N}, t\right) \mathrm{d} \mathscr{C}_{N} \mathrm{~d} \mathscr{V}_{N}=N !
$$


Flow parameters associated with the bubbles such as the mean number density $n(x, t)$ and the mean bubble velocity $\bar{v}(\mathbf{x}, t)$ are consequently defined as (Hirschfelder et al. 1954)

$$
n(\mathbf{x}, t)=\frac{1}{N !} \iint \sum_{k=1}^{N} \delta\left(\mathbf{x}_{k}-\mathbf{x}\right) f\left(\mathscr{C}_{N}, \mathscr{V}_{N}, t\right) \mathrm{d} \mathscr{C}_{N} \mathrm{~d} \mathscr{V}_{N}
$$

and

$$
n(\mathbf{x}, t) \overline{\mathbf{v}}(\mathbf{x}, t)=\frac{1}{N !} \iint \sum_{k=1}^{N} \mathbf{v}_{k} \delta\left(\mathbf{x}_{k}-\mathbf{x}\right) f\left(\mathscr{C}_{N}, \mathscr{V}_{N}, t\right) \mathrm{d} \mathscr{C}_{N} \mathrm{~d} \mathscr{V}_{N} .
$$

The void fraction $\epsilon(\mathbf{x}, t)$ is then defined as

$$
c(\mathbf{x}, t)=\frac{4}{3} \pi a^{3} n(\mathbf{x}, t)
$$

where $a$ is the radius of the bubbles. Also, the mean velocity of the dispersion is given by

$$
\overline{\mathbf{u}}(\mathbf{x}, t)=\frac{1}{N !} \iint \mathbf{u}(\mathbf{x}, t) f\left(\mathscr{C}_{N}, \mathscr{V}_{N}, t\right) \mathrm{d} \mathscr{C}_{N} \mathrm{~d} \mathscr{V}_{N},
$$

where $\mathbf{u}(\mathbf{x}, t)$ is the velocity at a point $\mathbf{x}$ at time $t$, irrespective of whether $\mathbf{x}$ is in a bubble or in the fluid.

Use shall also be made of an averaging of a bubble parameter $\mathbf{G}$ that is not an explicit function of time, with respect to a reference sphere being at $x_{0}$ having velocity $v_{0}$ in a dispersion consisting of $N+1$ spheres, i.e.

$$
\mathbf{G}\left(\mathbf{x}_{0}, t\right)=\int \mathrm{d} \mathbf{v}_{0} f\left(\mathbf{x}_{0}, \mathbf{v}_{0}, t\right)\left[\frac{1}{N !} \iint \mathbf{G}\left(\mathbf{x}_{0}, \mathbf{v}_{0}, \mathscr{C}_{N}, \mathscr{V}_{N}\right) f\left(\mathscr{C}_{N}, \mathscr{V}_{N}, t \mid \mathbf{x}_{0}, \mathbf{v}_{0}\right) \mathrm{d} \mathscr{C}_{N} \mathrm{~d} \mathscr{V}_{N}\right]
$$

Here $f\left(\mathscr{B}_{N}, \mathscr{V}_{N}, t \mid \mathbf{x}_{0}, \mathbf{v}_{0}\right)$ is the conditional pdf for finding at time $t$ spheres at $\mathbf{x}_{k}$ with velocity $\mathbf{v}_{k}$ $(k=1,2, \ldots, N)$ when it is given that there is a sphere at $\mathbf{x}_{0}$ with velocity $\mathbf{v}_{0}$.

In the following sections we shall adopt methods of analysis used in suspension rheology and in the study of the thermal properties of composite materials. In a Stokes flow problem, e.g. the sedimentation of small particles, the instantaneous velocities of the particles in an element of a suspension are uniquely specified by their instantaneous positions when the value of either the velocity or the force exerted per unit area at the boundary of the element is given (cf. Batchelor 1972). Thus, the ensemble averaging merely consists of averaging over a configurational probability distribution $P\left(\mathscr{C}_{N}\right)$. In contrast, in bubble flows hydrodynamic interaction between the bubbles gives rise to dynamic pressure forces of non-linear character; and here the use of the full pdf $f\left(\mathscr{C}_{N}, \mathscr{V}_{N}\right)$ will be required in general. An exception however, is the flow problem considered in section 3.1 , where the boundary conditions uniquely specify the potential flow field and the sphere velocities.

As mentioned above, in section 3.2 we shall assume the bubbles to have equal velocities. So here as well as the averaging reduces to an averaging over the probability of relative sphere positions; with the convenient consequence that for a contraction to a two-sphere problem the renormalization technique of Batchelor, developed for the Stokes flow problem (Batchelor 1972) and employed by Jeffrey (1973) for the thermal conductivity problem, can be applied. The mean value of $\mathbf{G}$ is then defined by

$$
\mathbf{G}\left(\mathbf{x}_{0}, t\right)=\frac{1}{N !} \int \mathbf{G}\left(\mathbf{x}_{0}, \mathscr{C}_{N}\right) P\left(\mathscr{C}_{N}, t \mid \mathbf{x}_{0}\right) \mathrm{d} \mathscr{C}_{N},
$$

where $P\left(\mathscr{C}_{N}, t \mid \mathbf{x}_{0}\right) d \mathscr{C}_{N}$ is the probability of finding at time $t, N$ spheres in the volume elements $\mathrm{d} \mathscr{C}_{N}$, given that there is a sphere at $\mathbf{x}_{0}$. In the following, reference to time $t$ shall be suppressed.

It is common practice in the two-phase flow literature to use the mean velocity of the fluid as a reference velocity for the description of the relative motion of the gas bubbles. In engineering practice the mean fluid velocity is an important parameter and of course one is free to choose any reference velocity that best meets one's purposes, however if one wants to adopt concepts developed in the classical literature for the motion of single bodies in unbounded flow fields the use of the mean velocity of the dispersion $\overline{\mathbf{u}}$ is conceptually more advantageous. Part of the reason is that a single rigid body experiences no force if it moves with the velocity of the flow at infinity, 
considered to be set up by some unspecified external mechanism. Therefore, forces exerted by the fluid as a consequence of the body's relative motion, e.g. due to a buoyancy force, are expressed in terms of the difference between the body's velocity and the fluid velocity at infinity. In the same manner the mean velocity of a dispersion may be considered to be specified, for instance by the volume fluxes of the two phases that are introduced into the bottom of a vertical pipe section. Again the bubbles would experience no force if they were imagined to move along with the mean velocity of the dispersion. So it seems to be more appropriate, in analogy to what one usually does in suspension rheology, to take this velocity as a reference velocity for the motion of the bubbles. The advantages of this method of description will become clear in the following sections.

\section{DILUTE DISPERSIONS}

\subsection{The Change of the Mean Bubble Velocity Due to an Instantaneous Acceleration of the Dispersion}

Consider an element of a bubbly dispersion in which the void fraction $\epsilon$, the mean bubble $\overline{\mathbf{v}}$ and the mean velocity $\overline{\mathbf{u}}$ may be taken to be statistically stationary functions of position. Imagine the element to be acted upon by impulsive forces such that the mean velocity is instantaneously changed by an amount $\Delta \overline{\mathbf{u}}$; we may then ask by what amount $\Delta \overline{\mathbf{v}}$ the mean bubble velocity instantaneously changes as a consequence. The answer may be given by the following line of reasoning (cf. Lighthill 1986).

In each realization of the dispersion the applied impulsive forces result in an impulsive excess pressure $p_{\mathrm{e}}$ in the fluid which exerts an impulsive force on each bubble $k$, given by

$$
-\int_{1}^{t+:} \mathrm{d} t \int p_{\mathrm{e}} \mathbf{n}_{k} \mathrm{~d} A \text {. }
$$

Here $\mathrm{d} A$ is a surface element coinciding with the bubble surface, $\mathbf{n}_{k}$ is the unit normal directed into the fluid and $\tau$ is the small time of action of the applied impulsive forces. Under the assumption that the bubbles are massless (an assumption that does not affect the final result for the added mass coefficient, which is associated with the fluid inertia), the impulsive force exerted by the fluid on each bubble equals zero, giving

$$
-\int \mathbf{n}_{k} \mathrm{~d} A \int_{t}^{t+\tau} p_{\mathrm{e}} \mathrm{d} t=0
$$

Furthermore, the excess pressure may be associated with the local change of the momentum of a particle of fluid, which makes it possible to define a potential such that

$$
\Delta \mathbf{u}(\mathbf{x})=\operatorname{grad} \phi, \quad \phi=-\frac{1}{\rho_{1}} \int_{1}^{t+\tau} p_{\mathrm{c}} \mathrm{d} t
$$

where $\phi$ satisfies the Laplace equation on account of the incompressibility of the fluid. Applying [4] yields, again on each bubble $k$,

$$
\int \phi \mathbf{n}_{k} \mathrm{~d} A=0
$$

Without the spheres the change of velocity would be $\Delta \overline{\mathbf{u}}$ throughout the fluid, so the velocity potential may be written as $\phi=\phi_{0}+\phi_{1}$, where $\phi_{0}=\Delta \overline{\mathbf{u}} \cdot \mathbf{x}$ and $\phi_{1}$ is the disturbance potential due to the motion of the bubbles. This definition of the potential, together with condition [5] and the condition

$$
\nabla \phi \cdot \mathbf{n}_{k}=\Delta \mathbf{v}_{k} \cdot \mathbf{n}_{k},
$$

uniquely determines the velocity potential and ensures that the change in velocity of each of the bubbles in any realization is given when the positions of the bubbles are specified.

It follows that the change in the mean velocity of the bubbles can be determined by concentrating on a reference sphere and ensemble averaging over the conditional probability distribution of the relative sphere positions $P\left(\mathscr{C}_{N} \mid \mathbf{x}_{0}\right)$, viz.

$$
\Delta \bar{v}=\frac{1}{N !} \int \Delta \mathbf{v}_{0}\left(\mathbf{x}_{0}, \mathscr{C}_{N}\right) P\left(\mathscr{C}_{N} \mid \mathbf{x}_{0}\right) \mathrm{d} \mathscr{C}_{N}
$$


Also, this change will be independent of the initial distribution of bubble velocities before the instantaneous acceleration, and therefore the change in the mean bubble velocity is the same as that of a dispersion which is initially at rest.

It is the latter situation that is envisaged in van Wijngaarden (1976) for the determination of the added mass coefficient of a dilute bubbly dispersion. van Wijngaarden finds, for details of the analysis the reader is referred to his paper,

$$
\Delta \overline{\mathbf{v}}=3(1-1.85 \mathrm{c})(\Delta \overline{\mathbf{u}}),
$$

correct to first order in the void fraction, i.e. the approximation in which only pair interactions are taken into account. Here it is assumed that the configurational pair pdf has the form

$$
P\left(\mathbf{x}_{0}+\mathbf{r} \mid \mathbf{x}_{0}\right)=\left\{\begin{array}{ll}
0, & r \leqslant 2 a, \\
n\left(\mathbf{x}_{0}+\mathbf{r}\right)=n\left(\mathbf{x}_{0}\right), & r>2 a,
\end{array}\right\}
$$

expressing that no spheres can overlap, that the probability of finding a sphere at $\mathbf{x}_{0}+\mathbf{r}$ is independent of whether there is a second sphere at $x_{0}$ and that the dispersion is spatially homogeneous in the considered element around $\mathbf{x}_{0}$.

The result can be associated with an added mass coefficient by reasoning as follows (cf. Landau $\&$ Lifshitz 1959). When the bubbles "move along" with the fluid the impulsive force density on the volume taken in by the bubbles would be

$$
\frac{4}{3} \pi a^{3} n \rho_{1} \Delta \overline{\mathbf{u}}=\epsilon \rho_{1} \Delta \overline{\mathbf{u}} .
$$

The total impulsive force density on the bubbles consists of this contribution and a contribution which accounts for the additional momentum to be transmitted to the fluid in order to accelerate the bubbles relative to the mean velocity of the dispersion. Writing this latter contribution as $m(\epsilon)(\Delta \overline{\mathbf{v}}-\Delta \bar{u})$, the condition that the total impulsive force on the bubbles is equal to zero then gives

$$
c \rho_{1} \Delta \overline{\mathbf{u}}-m(c)(\Delta \overline{\mathbf{v}}-\Delta \overline{\mathbf{u}})=0 .
$$

Combining [6] and [8] the added mass coefficient $m(\epsilon)$, thus defined, is found to be

$$
m(\epsilon)=\frac{1}{2} \rho_{1} \epsilon(1+2.78 \epsilon)+O\left(\epsilon^{3}\right) .
$$

The contribution of $O\left(\epsilon^{2}\right)$ accounts for the pairwise interaction of the spheres. In the absence of interactions the added mass of each of the spheres is $\frac{1}{2} \rho_{1}\left(\frac{4}{3} \pi a^{3}\right)$ and we would have $m(\epsilon)=\frac{1}{2} \rho_{1} \epsilon$.

\subsection{The Mean Fluid Impulse}

Consider as before a dispersion of equally-sized rigid spherical bubbles in a fluid; the number density is $n(\mathbf{x}, t)$ and the mean bubble velocity and the mean velocity of the dispersion are given by $\overline{\mathbf{v}}(\mathbf{x}, t)$ and $\overline{\mathbf{u}}(\mathbf{x}, t)$, respectively. Assume that there is a region about $\mathbf{x}$, of volume $V$ and consisting of a large number $(N+1)$ spheres, within which the flow is approximately uniform. When the spheres are carried along with the fluid the mean momentum of the fluid outside the spheres is given by $\rho_{1}(1-\epsilon) \overline{\mathbf{u}}$. The mean fluid impulse I may then be defined [in the sense of Landau \& Lifschitz (1959)] as the ensemble mean of the additional momentum that is transmitted to the fluid when the bubbles are instantaneously (through the action on the bubbles of hypothetical large impulsive forces working over a short time interval) given the velocities that, at the moment of consideration, characterize each member of the ensemble of realizations of the flow. On account of the equivalence of ensemble averaging and volume averaging for a homogeneous flow, it is given by

$$
n \mathbf{I}=\frac{1}{V} \sum_{k} \mathbf{I}_{k} \quad(k=0,1,2, \ldots, N)
$$

with

$$
\mathbf{I}_{k}=\rho_{1} \int \phi_{1} \mathbf{n}_{k} \mathrm{~d} A
$$


Here $\phi_{1}$ is the velocity potential associated with this instantaneous relative acceleration. The potential is uniquely determined by the specification of the sphere velocities and the boundary conditions

$$
\nabla \phi_{1} \cdot \mathbf{n}_{k}=\left(\mathbf{v}_{k}-\overline{\mathbf{u}}\right) \cdot \mathbf{n}_{k} .
$$

The analysis is somewhat easier when first use is made of the relation between the impulse $\mathbf{I}_{k}$ and the dipole strength $\mathbf{G}_{k}$ of the image system of sphere $k$ associated with the change in the velocity of the fluid (Lighthill 1986)

$$
\mathbf{I}_{k}=\rho_{1} \mathbf{G}_{k}-\frac{4}{3} \pi a^{3} \rho_{1}\left(\mathbf{v}_{k}-\overline{\mathbf{u}}\right) .
$$

We then have

$$
n \mathbf{I}=-\rho_{1} c(\overline{\mathbf{v}}-\overline{\mathbf{u}})+\rho_{1} \frac{1}{V} \sum_{k} \mathbf{G}_{k} .
$$

When the dispersion is so dilute that the effects of hydrodynamic interactions are negligibly small, each sphere may be assumed to be alone in an infinite fluid and the mean dipole strength will be given by

$$
\mathbf{G}_{0}=2 \pi a^{3}(\overline{\mathbf{v}}-\overline{\mathbf{u}}),
$$

with the familiar result

$$
n \mathbf{I}_{0}=\frac{1}{2} \rho_{1} \epsilon(\overline{\mathbf{v}}-\overline{\mathbf{u}})
$$

This allows us to write [10] as

$$
n \mathbf{I}=\frac{1}{2} \rho_{1} \epsilon(\overline{\mathbf{v}}-\overline{\mathbf{u}})+\rho_{1} \frac{1}{V} \sum_{k} \mathbf{G}_{1 k} .
$$

Here $\mathbf{G}_{1 k}=\mathbf{G}_{k}-\mathbf{G}_{0}$ is the additional dipole strength of sphere $k$ due to the presence of the neighbouring spheres.

Next, take the steps of concentrating on a reference sphere, with velocity $\mathbf{v}_{0}$ at $\mathbf{x}_{0}$ say, and converting the averaging into an ensemble averaging

$$
\frac{1}{V} \sum_{k} \mathbf{G}_{1 k}=n \mathbf{G}_{1}
$$

with

$$
\mathbf{G}_{1}=\int \mathrm{d} \mathbf{v}_{0} f\left(\mathbf{x}_{0}, \mathbf{v}_{0}\right)\left[\frac{1}{N !} \iint \mathbf{G}_{1}\left(\mathbf{x}_{0}, \mathbf{v}_{0}, \mathscr{C}_{N}, \mathscr{V}_{N}\right) f\left(\mathscr{C}_{N}, \mathscr{V}_{N} \mid \mathbf{x}_{0}, \mathbf{v}_{0}\right) \mathrm{d} \mathscr{B}_{N} \mathrm{~d} \mathscr{V}_{N}\right]
$$

No progress can now be made without knowledge about the probability distribution of the spheres. In the two-phase flow literature the sphere velocities are usually taken to be equal to the mean bubble velocity $\overline{\mathbf{v}}$ in order to make an analogy possible with the thermal conductivity problem, and we shall continue the analysis upon this assumption. The averaging then reduces to an averaging over the configuration of the sphere positions relative to that of the reference sphere, viz.

$$
\mathbf{G}_{1}=\frac{1}{N !} \int \mathbf{G}_{1}\left(\mathbf{x}_{0}, \mathscr{C}_{N}\right) P\left(\mathscr{C}_{N} \mid \mathbf{x}_{0}\right) d \mathscr{C}_{N} .
$$

In the case of dilute dispersions the natural way of obtaining an approximate expression for the mean dipole strength would be to evaluate [12] by allowing for pair interactions between the reference sphere and one other sphere only:

$$
\mathbf{G}_{1}=\int \mathbf{G}_{1}\left(\mathbf{x}_{0}, \mathbf{x}_{0}+\mathbf{r}\right) \boldsymbol{P}\left(\mathbf{x}_{0}+\mathbf{r} \mid \mathbf{x}_{0}\right) \mathrm{d} \mathbf{r} .
$$


However, this procedure would lead to a conditionally convergent integral. The problem does not arise when the renormalization technique devised by Batchelor (1972) is applied first. When the reference sphere is replaced by fluid the mean velocity at $x_{0}$ is given by

$$
\frac{1}{N !} \iint \mathbf{u}\left(\mathbf{x}_{0}, \mathscr{C}_{N}, \mathscr{V}_{N}\right) f\left(\mathscr{C}_{N}, \mathscr{V}_{N}\right) \mathrm{d} \mathscr{C}_{N} \mathrm{~d} \mathscr{V}_{N}=\overline{\mathbf{u}}
$$

or, when the spheres are assumed to have equal velocities

$$
\frac{1}{N !} \int \mathbf{u}\left(\mathbf{x}_{0}, \mathscr{C}_{N}\right) P\left(\mathscr{C}_{N}\right) \mathrm{d} \mathscr{C}_{N}=\overline{\mathbf{u}}
$$

Therefore, we may write

$$
\frac{1}{N !} \int \nabla \phi_{1}\left(\mathrm{x}_{0}, \mathscr{C}_{N}\right) P\left(\mathscr{C}_{N}\right) \mathrm{d} \mathscr{C}_{N}=0
$$

Adding $2 \pi a^{3}$ times this expression to [12], gives

$$
\overline{\mathbf{G}}_{1}=\frac{1}{N !} \int\left[\mathbf{G}_{1}\left(\mathbf{x}_{0}, \mathscr{C}_{N}\right) P\left(\mathscr{C}_{N} \mid \mathbf{x}_{0}\right)+2 \pi a^{3} \nabla \phi_{1}\left(\mathbf{x}_{0}, \mathscr{C}_{N}\right) P\left(\mathscr{C}_{N}\right)\right] \mathrm{d} \mathscr{C}_{N},
$$

and upon reduction to the two-sphere approximation we now obtain

$$
\mathbf{G}_{1}=\frac{1}{N !} \int\left[\mathbf{G}_{1}\left(\mathbf{x}_{0}, \mathbf{x}_{0}+\mathbf{r}\right) P\left(\mathbf{x}_{0}+\mathbf{r} \mid \mathbf{x}_{0}\right)+2 \pi a^{3} \nabla \phi_{1}\left(\mathbf{x}_{0}, \mathbf{x}_{0}+\mathbf{r}\right) P\left(\mathbf{x}_{0}+\mathbf{r}\right)\right] \mathrm{d} \mathbf{r},
$$

an integral that is absolutely convergent, as we shall see; [14] may be compared with expression [3.13] in Jeffrey (1973), where it is shown that it is correct to $O\left(\epsilon^{2} / n\right)$.

For the calculation of [14] we shall take

$$
\begin{aligned}
P\left(\mathrm{x}_{0}+\mathrm{r}\right) & =P\left(\mathrm{x}_{0}\right)=n\left(\mathrm{x}_{0}\right), \\
P\left(\mathbf{x}_{0}+\mathbf{r} \mid \mathbf{x}_{0}\right) & =\left\{\begin{array}{ll}
0, & r \leqslant 2 a, \\
n\left(\mathrm{x}_{0}\right), & r>2 a,
\end{array}\right\}
\end{aligned}
$$

a natural choice, having made the assumption of equal sphere velocities (cf. [7]). The velocity at $\mathbf{x}_{0}$, relative to $\mathfrak{a}$, due to a single sphere at $\mathbf{x}_{0}+r$ is given by

$$
\nabla \phi_{1}\left(\mathbf{x}_{0}, \mathbf{x}_{0}+\mathbf{r}\right)=\left\{\begin{array}{ll}
\overline{\mathbf{v}}-\overline{\mathbf{u}}, & r \leqslant 2 a, \\
-\frac{(\overline{\mathbf{v}}-\overline{\mathbf{u}}) a^{3}}{2 r^{3}}+\frac{3(\overline{\mathbf{v}}-\overline{\mathbf{u}}) \cdot r a^{3}}{2 r^{5}} \mathbf{r}, & r>2 a,
\end{array}\right\}
$$

and the additonal dipole strength may be inferred directly from Jeffrey's (1973) solution of the two-sphere problem:

$$
\mathbf{G}_{1}=2 \pi a^{3}\left[\frac{(\overline{\mathbf{v}}-\tilde{\mathbf{u}}) a^{3}}{2 r^{3}}+\frac{3(\overline{\mathbf{v}}-\tilde{\mathbf{u}}) \cdot \mathbf{r} a^{3}}{2 r^{5}} \mathbf{r}\right]-2 \pi a^{3} \sum_{p=6}^{\infty}\left(\frac{a}{r}\right)^{p}\left[A_{p}(\overline{\mathbf{v}}-\tilde{\mathbf{u}})-B_{p} \frac{(\overline{\mathbf{v}}-\tilde{\mathbf{u}}) \cdot \mathbf{r}}{r^{2}} \mathbf{r}\right] .
$$

Values for the coefficients $A_{p}$ and $B_{p}$ may be found in his paper. Note that the term in square brackets in [17] is precisely $-\nabla \phi_{1}\left(\mathbf{x}_{0}, \mathbf{x}_{0}+\mathbf{r}\right)$ for $r>2 a$; it is this term which renders the integral in [13] conditionally convergent, but which is removed by application of Batchelor's renormalization technique.

After subsitution of [15]-[17] into [14] it can be shown that the mean additional dipole strength results from two contributions,

$$
\mathbf{G}_{1}=2 \pi a^{3} n \int_{r<a}(\overline{\mathbf{v}}-\mathbf{u}) \mathrm{d} \mathbf{r}-2 \pi a^{3} n \int_{r>2 a}\left\{\sum_{p=6}^{\infty}\left(\frac{a}{r}\right)^{p}\left[A_{p}(\overline{\mathbf{v}}-\overline{\mathbf{u}})-B_{p} \frac{(\overline{\mathbf{v}}-\overline{\mathbf{u}}) \cdot \mathbf{r}}{r^{2}} \mathbf{r}\right]\right\} \mathrm{d} \mathbf{r} .
$$

Evaluation of the integrals shows the first contribution to be

$$
\frac{3}{2}\left(\frac{4}{3} \pi a^{3}\right) \epsilon(\overline{\mathbf{v}}-\overline{\mathbf{u}})
$$


whereas the second contribution is

$$
\frac{3}{2}\left(\frac{4}{3} \pi a^{3}\right) \epsilon(\overline{\mathbf{v}}-\overline{\mathbf{u}})\left[\sum_{p=6}^{x} \frac{B_{p}-3 A_{p}}{(p-3) 2^{p-3}}\right] .
$$

The final expression for the fluid impulse density is given by

$$
n \mathbf{I}=\frac{1}{2} \rho_{1} \epsilon(\overline{\mathbf{v}}-\overline{\mathbf{u}})\left\{1+3 \epsilon\left[1+\sum_{p=6}^{x} \frac{B_{p}-3 A_{p}}{(p-3) 2^{p-3}}\right]\right\}
$$

or, after numerical evaluation of the summation,

$$
n \mathbf{I}=\frac{1}{2} \rho_{1} \epsilon(1+3.32 \epsilon)(\overline{\mathbf{v}}-\overline{\mathbf{u}}) .
$$

It may be associated with an added mass coefficient $m(\epsilon)$ by writing

$$
n \mathbf{I}=m(\epsilon)(\overline{\mathbf{v}}-\overline{\mathbf{u}})
$$

from which it follows that

$$
m(c)=\frac{1}{2} \rho_{1} c(1+3.32 \epsilon)
$$

It is instructive to compare the expression for the mean additional dipole strength [18], viz.

$$
n \mathbf{G}_{1}=\frac{3}{2} \epsilon^{2}\left[+\sum_{p=6}^{\infty} \frac{B_{p}-3 A_{p}}{(p-3) 2^{p-3}}\right](\overline{\mathbf{v}}-\overline{\mathbf{u}})
$$

with that of Jeffrey (1973) for the mean additional thermal dipole strength of non-conducting spheres $\left(\beta=-\frac{1}{2}\right.$ in that paper),

$$
n \mathbf{S}_{1}=-\frac{3}{2} \epsilon^{2}\left[-\frac{1}{2}+\sum_{p=6}^{x} \frac{B_{p}-3 A_{p}}{(p-3) 2^{p-3}}\right](\nabla T)
$$

The results may be compared when replacing $(\overline{\mathbf{v}}-\tilde{\mathbf{u}})$ by $(-\nabla \bar{T})$. The contributions associated with the integration over the range $r>2 a$ in [18] appear to be identical, but those arising from the integration over $r \leqslant a$ are different. The reason is that the potential field inside a single sphere does not have the same form, namely $(\overline{\mathbf{v}}-\overline{\mathbf{u}}) \cdot \mathbf{x}$ in the fluid dynamical problem, and $\frac{3}{2}(\nabla T) \cdot \mathbf{x}$ in the thermal conductivity problem. This shows that even with the assumption that all spheres have equal velocities, the two problems are essentially different. The potential fields outside the spheres may be compared because they are determined by similar conditions on the normal derivatives of the potential at the surfaces of the spheres. However, in the thermal conductivity problem we have the additional condition of continuity of the potential on the sphere surfaces, a condition not imposed in the fluid dynamical problem. That the potential field inside the spheres is relevant for the calculation of the fluid impulse, which according to [10] seems to be determined by the potential field of the fluid outside the spheres can be understood from the following argument (Jeffrey 1973, section 6).

To obtain the very dilute approximation [11] it was assumed that each sphere moved with a velocity $\overline{\mathbf{v}}-\overline{\mathbf{u}}$ in a fluid at rest at infinity. The volume flow associated with the bubble motion is $c(\overline{\mathbf{v}}-\overline{\mathbf{u}})$ and therefore the average fluid is $-c(\overline{\mathbf{v}}-\overline{\mathbf{u}})+O\left(\epsilon^{2}\right)$ in order to maintain zero mean volume flux. So a second-order correction to [10] can be obtained by supposing that each of the spheres is alone in an infinite medium with velocity $-\epsilon(\overline{\mathbf{v}}-\overline{\mathbf{u}})$ at infinity. The dipole strength of each of the spheres is then

$$
\mathbf{G}=2 \pi a^{3}(\overline{\mathbf{v}}-\overline{\mathbf{u}})(1+\epsilon)
$$

and the impulse density is

$$
n \mathbf{T}=\frac{1}{2} \rho_{1} \epsilon(1+3 \epsilon)(\overline{\mathbf{v}}-\overline{\mathbf{u}}) .
$$

If the velocity field inside the spheres were different the second-order correction would have differed correspondingly. Note that the argument leading to [20] makes no reference to any assumption on the bubble velocities.

We are now in the position to explain the differences between the results of van Wijngaarden (1976), Zuber (1964) and [19]. The contribution $\frac{3}{2} \rho_{1} \epsilon^{2}$ to the added mass coefficient is simply the 
result of the presence around each of the bubbles of neighbouring bubbles. The precise value of the $O\left(\epsilon^{2}\right)$-term depends on the details of the interactions between the bubbles in the dilute limit, and therefore depends on the form of the pair probability distribution of sphere positions and velocities. This velocity distribution follows in van Wijngaarden (1976) from the condition that in each realization the velocity change of the bubbles should be such that no additional momentum is transmitted to the fluid by each of the bubbles, while in this section we determined the additional momentum that should be transmitted in order to set up a specific velocity distribution, viz. one in which in each of the realizations the bubbles have equal velocities $\overline{\mathbf{v}}$.

In a dispersion where all the spheres are far removed from each other the distance between neighbouring spheres is $O\left(a \epsilon^{-1.3}\right)$ and so the probability of finding a sphere around any other sphere within the range $r<a \epsilon^{-1: 3}$ is zero and is $O(n)$ for $r>a \epsilon^{-1: 3}$. An approximate expression for the added mass of a dilute dispersion may therefore be determined by assuming a massless sphere to be located at the centre of another sphere with radius $a \epsilon^{-1 ; 3}$. Whether the velocity is calculated that the inner sphere would obtain upon an instantaneous acceleration of the entire system from rest to a velocity $\overline{\mathbf{u}}$, or the fluid impulse when the inner sphere has velocity $\overline{\mathbf{v}}$ and the outer sphere velocity $\overline{\mathbf{u}}$, the same result for the added mass will be found. This can be inferred from Milne-Thomson (1968):

$$
m(\epsilon)=\frac{1}{2} \rho_{1} \epsilon\left(\frac{1+2 \epsilon}{1-\epsilon}\right)
$$

The method sketched above is the one used by Zuber (1964); for small $c$ we have

$$
m(\epsilon)=\frac{1}{2} \rho_{1} \epsilon(1+3 \epsilon)+O\left(\epsilon^{3}\right) \text {. }
$$

\section{NON-DILUTE DISPERSIONS}

\subsection{Periodic Arrays}

An exact expression for the added mass coefficient, valid for any value of the void fraction, can be found by assuming the bubbles to be arranged in an infinitely extended spatially periodic array. By symmetry, the change in velocity upon an instantaneous acceleration of the dispersion has the same value for each of the spheres and is therefore equal to the change in the mean velocity. Furthermore, it is clear that the added mass coefficient found by a determination of this velocity change is equal to that calculated from the mean fluid impulse if it is assumed that in the latter case the spheres have equal velocities. By the arguments given in section 3.1 we can address the problem by calculating the velocity of the spheres when accelerated instantaneously from rest, and we then have to solve the Laplace equation with the boundary condition $\nabla \phi \cdot \mathbf{n}=\overline{\mathbf{v}} \cdot \mathbf{n}$ on each of the spheres, and the further condition that the mean velocity in a unit cell of the array takes the value $\overline{\mathbf{u}}$. The mean bubble velocity subsequently follows from the condition that for each of the spheres

$$
\int \phi \mathbf{n d} \mathbf{A}=0
$$

This potential flow problem is essentially analogous to the problem of determining the effective thermal conductivity of a composite material consisting of periodically arranged non-conducting spheres in a homogeneous matrix, for which a method of solution has been developed by Sangani $\&$ Acrivos $(1982,1983)$; their method will be adopted here.

Formal solution of the potential flow problem

Assume that the positions of the centres of the spheres are given by

$$
\mathbf{r}_{n}=n_{1} \mathbf{a}_{1}+n_{2} \mathbf{a}_{2}+n_{3} \mathbf{a}_{3} \quad\left(n_{1}, n_{2}, n_{3}=0, \pm 1, \pm 2, \ldots\right)
$$

where $a_{1}, a_{2}$ and $a_{3}$ are the basic vectors determining the unit cell of the array. The three cubic arrays examined in what follows are: simple cubic (SC), body-centred cubic (BCC) and face-centred cubic (FCC); the reader may find expressions for the basic vectors of these arrangements in appendix 1 of Sangani \& Acrivos (1982). Taking for simplicity the mean motion 
of the fluid and the bubbles to be directed along the $x_{1}$-axis, the fluid potential can then be expressed as

$$
\phi=u^{*} x_{1}+\phi
$$

where $\phi$ is a spatially periodic function

$$
\phi(\mathbf{r})=\phi\left(\mathbf{r}+\mathbf{r}_{n}\right)
$$

with $\mathbf{r}_{n}$ given by [23]. The as yet unknown velocity $u^{*}$ will be specified later from the condition that the mean velocity in a unit cell must equal $\bar{u}$.

A formal solution for the potential field $\phi$ reads

$$
\phi(\mathbf{r})=\mathbf{G} S_{1},
$$

where

$$
S_{1}=\frac{1}{\pi \tau_{0}} \sum_{n} \cdot \frac{\mathrm{e}^{-2 \pi i}\left(\mathbf{k}_{n} \cdot \mathbf{r}\right)}{k_{n}^{2}}
$$

and $\mathbf{G}$ is the differential operator

$$
\mathbf{G}=\sum_{m+n+p=1}^{x}\left(u^{*}-\bar{v}\right) A_{m n p} \frac{\partial^{m+n+p}}{\partial x_{1}^{m} \partial x_{2}^{n} \partial x_{3}^{p}} \quad\left(\mathbf{r}=x_{1}, x_{2}, x_{3}\right) .
$$

Here $\tau_{0}$ is the volume of a unit cell of the array and $k_{n}$ are the reciprocal lattice vectors given by

$$
\mathbf{k}_{n} \cdot \mathbf{a}_{j}=n_{j} \quad(j=1,2,3) .
$$

Note that [25]-[27] are only valid outside the spheres; inside the spheres the flow field is given by the constant mean bubble velocity $v$. The coefficients $A$ in [27] subsequently follow from the condition that the normal components of the fluid velocity and bubble velocity are equal on the surfaces of the bubbles.

Inside the unit cell containing the origin the fluid potential $\phi$ can also be represented as a sum of spherical harmonics:

$$
\phi=\sum_{n=1}^{x} \sum_{m=1}^{m \leq 1 \cdot 2 n}\left(u^{*}-\bar{\imath}\right)\left(E_{n m}+F_{n m} r^{1-4 n}\right) Y_{2 n-1}^{4 m}\left(x_{1}, x_{2}, x_{3}\right)
$$

where

$$
Y_{n}^{m}\left(x_{1}, x_{2}, x_{3}\right)=r^{n} P_{n}^{m}(\cos \theta) \cos m \varphi
$$

and

$$
x_{1}=r \cos \theta, \quad x_{2}=r \sin \theta \cos \varphi, \quad x_{3}=r \sin \theta \sin \varphi .
$$

Application of the forementioned boundary condition yields the relation

$$
F_{n m 1}+L_{n} a^{4 n-1} E_{n m}=-L_{n} a^{3} \delta_{1 n} \text {, }
$$

with $L_{n}$ given by

$$
L_{n}=-\frac{2 n-1}{2 n}
$$

By making use of the expansion of $S_{1}$ in spherical harmonics near $r=0$ from Hasimoto (1959),

$$
S_{1}=\frac{1}{r}-\bar{c}+\frac{2 \pi}{3 \tau_{0}} r^{2}+\sum_{n=2}^{x} \sum_{m=0}^{m \leq 1: 2 n} a_{n m 1} Y_{2 n}^{4 m}\left(x_{1}, x_{2}, x_{3}\right)
$$

where $\bar{c}$ and $a_{n m}$ are constants characteristic of the particular type of array, and by reformulating the differential operator as

$$
\mathbf{G}=\sum_{M=0}^{x} \sum_{m=0}^{m \leqslant 1 \cdot 2 M} \frac{2^{4 m-1}}{(2 n+1) !}\left(u^{*}-\bar{t}\right) A_{n m} \frac{\partial^{2 n+1}}{\partial x_{!}^{2 n+1}}\left[\left(\frac{\partial}{\partial \xi}\right)^{4 m}+\left(\frac{\partial}{\partial \eta}\right)^{4 m}\right],
$$

with

$$
M=n+2 m, \quad \xi=x_{2}+i x_{3}, \quad \eta=x_{2}-i x_{3},
$$


we are then able to establish, upon substituting [30] and [31] into [25], two additional relations between the coefficients $A_{n m}, E_{n m}$ and $F_{n m}$. Together with [29] these lead to a set of linear equations in the unknown $A_{n m}$, viz.

$$
\begin{array}{r}
A_{n m}=L_{m+1} \frac{a^{4 m+3}}{(2 M+4 m+1) !} \sum_{j=0}^{\infty} \sum_{j=0}^{j<1 / 2 J}\left[\frac{\lambda_{1}\left(2 p+4 q_{1}\right) !}{(2 J-4 j+1) !} a_{p, q_{1}}+\frac{\lambda_{2}\left(2 p+4 q_{2}\right) !}{(2 J-4 j+1) !} a_{p, q_{2}}\right] A_{J-2 j, j} \\
+L_{1} a^{3} \delta_{M .0}\left(1+\frac{4 \pi}{3 \tau_{0}} A_{00}\right)
\end{array}
$$

with

$$
\begin{gathered}
p=M+J+1, \quad q_{1}=m+j, \quad q_{2}=|m-j|, \\
\begin{cases}\lambda_{1}=\lambda_{2}=\frac{1}{4} & \text { if } m=0, j \neq 0, \\
\lambda_{1}=\frac{1}{2}, \quad \lambda_{2}=1 & \text { if } m=j \neq 0, \\
\lambda_{1}=\lambda_{2}=\frac{1}{2} & \text { otherwise. }\end{cases}
\end{gathered}
$$

The solution, for given $\bar{v}$ and $\bar{u}$, is finally completed by imposing the condition that the mean velocity in the unit cell equals $\bar{u}$, i.e.

$$
\frac{1}{\tau_{0}}\left(\frac{4}{3} \pi a^{3} \bar{v}+\int \nabla \phi \mathrm{d} V_{1}\right)=\bar{u}
$$

where the integration is over the volume taken in by the fluid. Note that a similar condition need not be posed on the temperature distribution in the unit cell of the temperature problem. If the imposed non-periodic temperature gradient is taken equal to the mean temperature gradient it is satisfied automatically. Evaluation of [32] yields

$$
\bar{u}=u^{*}+\frac{4 \pi}{\tau_{0}} A_{00}\left(u^{*}-v\right)
$$

\section{The added mass coefficient}

As explained above, the change in the mean bubble velocity follows from the condition on the surface of each sphere,

$$
\int \phi n_{1} \mathrm{~d} A=0
$$

where $n_{1}$ is the $x_{1}$-component of the unit normal directed into the fluid. This is equivalent to

$$
\frac{4}{3} \pi a^{3} \bar{v}+4 \pi A_{00}\left(\bar{v}-u^{*}\right)=0 .
$$

Combined with [33] this yields

$$
\bar{v}=\bar{u}\left[1+\epsilon\left(1+\frac{\tau_{0}}{4 \pi A_{00}}\right)\right]^{-1} .
$$

In the manner described at the end of section 3.1 an added mass coefficient $m(\epsilon)$ may then be defined, which is given by

$$
m(\epsilon)=-\rho_{1} c\left[1+\frac{1}{\epsilon\left(1+\frac{\tau_{0}}{4 \pi A_{00}}\right)}\right] .
$$

This expression has been evaluated numerically for the three types of arrays and the results, together with that of van Wijngaarden for a dilute (random) dispersion, are shown in figure 1. For the calculation of the constants $\bar{c}$ and $a_{n m}$ the direct sum method, described in appendix 1 of Sangani \& Acrivos (1982), was used and it was found that the computed values were in exact agreement with those given in table 4 of that paper. 


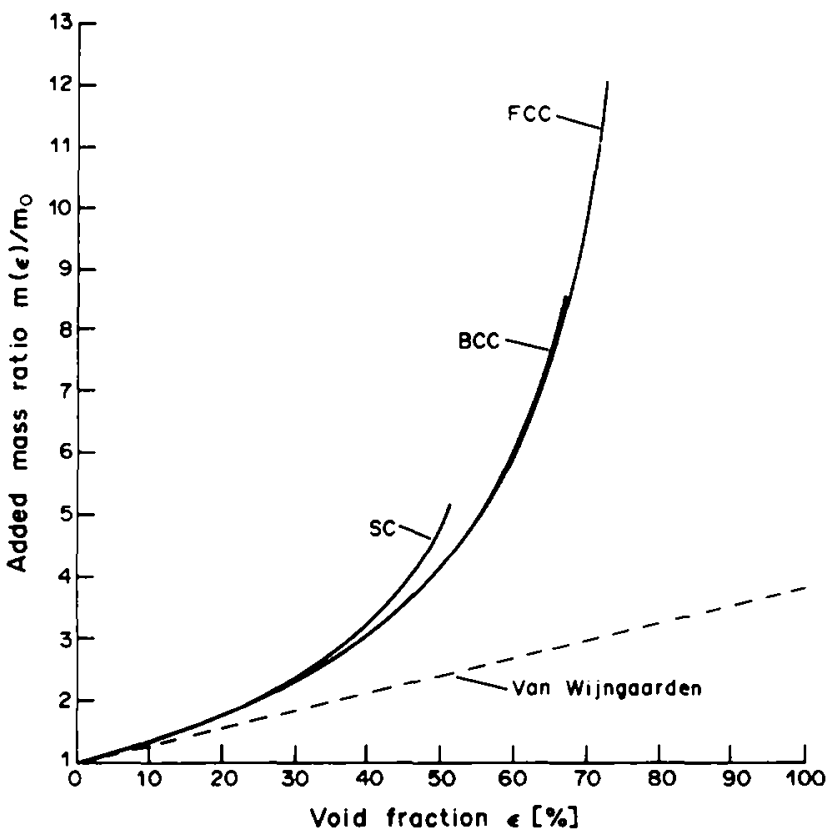

Figure. 1. Added mass, according to the definition of van Wijngaarden (1976), as a function of the void fraction for random dilute bubbly dispersions and dispersions with periodically arranged bubbles $\left(m_{0}=\frac{1}{2} \rho, \epsilon\right)$.

\subsection{Random Arrays}

An approximate expression for the added mass coefficient of a random non-dilute dispersion may be obtained through interpolation of the results for the three types of arrays found above. A fit suggested by the work of Sangani \& Acrivos (1983) is

$$
m(c)=\frac{1}{2} \rho_{1} \epsilon\left[K_{1} \ln \left(\epsilon_{\max }-\epsilon\right)+K_{2}\right],
$$

where $\epsilon_{\max }=0.62$ is the void fraction of a random closely packed dispersion. For $\epsilon \leqslant 0.35$ an excellent fit is achieved when we let the constants $K_{1}$ and $K_{2}$ take the value $K_{1} \approx \cdots-1.98$ and $K_{2} \approx 0.05$. For comparison, [36] and the other approximate relations [19] and [21] are shown in figure 2. Note that for $\epsilon \leqslant 0.35,[21]$ and [36] yield almost identical results.

\section{DISCUSSION}

After dealing with the relations published in the two-phase flow literature for the added mass coefficient, we discuss their applicability in the description of the average motion of gas bubbles.

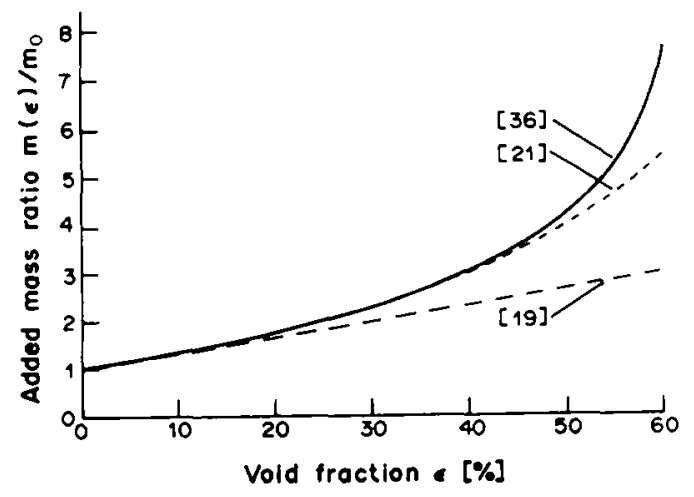

Figure 2. Added mass, defined by means of the fluid impulse, as a function of the void fraction for random dispersions of bubbles with equal velocities $\left(m_{0}=\frac{1}{2} \rho_{1} t\right)$. 
Such a theory is effectively a continuum theory, and it therefore underlies the usual assumption that the time scales and length scales of the motion of the dispersion are such that meaningful (observable) averages can be defined that are continuous space- and time-dependent field variables; here this implies that locally the bubbly dispersion should not be far removed from the uniform "equilibrium" state.

The dispersion studied by van Wijngaarden (1976) was instantaneously accelerated. As a consequence the system is, if it was in some type of equilibrium, instantaneously pushed out of this equilibrium. The result given for the change in the mean bubble velocity due to this instantaneous acceleration [9] is a fair approximation-it would be exact if it could be shown that the pair probability distribution [7] is representative for a real flow-and an added mass coefficient may of course be associated with it, but it is not the coefficient to be used in a continuum theory for the average bubble motion. The mean fluid impulse, and so the related added mass coefficient, is determined by the probability distribution of bubble positions and velocities that represents the state of the dispersion at a particular instant. For the dynamics of the flow it may be interpreted as an addition to the mean momentum of the gas of an element of the dispersion, in the sense that part of the mean force exerted by the fluid on the bubbles is expressible in terms of the time rate of change of this additional momentum. It is this concept of added mass that is of relevance to a continuum theory of the bubble motion. In a further study (Biesheuvel \& Gorissen 1989) this will be worked out in a derivation of the momentum conservation equation for the bubbles based on elements of the kinetic theory of dense gases, and which, together with a conservation equation for the mean bubble number density, will form the basis of a description of the propagation of void fraction disturbances in a uniform bubbly fluid.

In section 3.2 progress could only be made by assuming that in each realization of the flow the bubbles have equal velocities. van Wijngaarden's (1976) method, in which in each member of the ensemble the sphere velocities are different, yields an added mass coefficient $m(\epsilon)=\frac{1}{2} \rho_{1} c(1+2.78 \epsilon)$ that differs from $m(\epsilon)=\frac{1}{2} \rho_{1} \epsilon(1+3.32 \epsilon)$, obtained from the fluid impulse, so we suspect that the added mass coefficient, as calculated in section 3.2 , will be modified when velocity fluctuations from the mean are accounted for. The effect is only of $o\left(\epsilon^{2}\right)$, and in practice Zuber's (1964) results $m(\epsilon)=\frac{1}{2} \rho_{1} \epsilon(1+3 c)$ will be a valid approximation.

When the dispersion is non-dilute the importance of the velocity fluctuations is likely to be less and [36] or [21], viz.

$$
m(\epsilon)=\frac{1}{2} \rho_{1} c\left(\frac{1+2 \epsilon}{1-\epsilon}\right)
$$

may be used. Uniform bubbly flow does not occur in practice above values of the void fraction of about 0.3 , when this flow pattern is unstable to void fraction disturbances, however in a theory that explains this phenomenon the results may be of assistance.

The random motion of the gas bubbles is also the reason that the simple relationship that exists between the impulse and the kinetic energy of the fluid for the motion of a single rigid body is not found for the ensemble mean values of these quantities for a bubbly dispersion. The mean kinetic energy of the fluid at a point $\mathbf{x}$ in the dispersion can be determined in the same manner as the fluid impulse in the previous section, by assuming again that there is a region about the point $\mathbf{x}$ of volume $V$, in which the flow variables are statistically stationary random functions of position. When the spheres move along with the fluid the velocity field will be uniform with value $u$. The additional mean kinetic energy of the fluid due to the relative motion of the bubbles can be thought to be transmitted to the fluid by the action over a very short time $\tau$ of large impulsive forces $\mathbf{f}_{k}$ on the bubbles in order to set up this relative motion. These forces do work

$$
\int_{1}^{t+\tau} \frac{t}{\tau} \sum_{k}\left(\mathbf{v}_{k}-\overline{\mathbf{u}}\right) \cdot \mathbf{f}_{k} \mathrm{~d} t=\frac{1}{2} \sum_{k}\left(\mathbf{v}_{k}-\tilde{\mathbf{u}}\right) \cdot\left(\mathbf{f}_{k} \tau\right)
$$

or, equivalently,

$$
\frac{1}{2} \sum_{k}\left(\mathbf{v}_{k}-\overline{\mathbf{u}}\right) \cdot \mathbf{I}_{k}
$$


The mean additional kinetic energy density is thus given by

$$
\frac{1}{2} n \overline{(\mathbf{v}-\overline{\mathbf{u}}) \cdot \mathbf{I}}=\frac{1}{2} n(\overline{\mathbf{v}}-\overline{\mathbf{u}}) \cdot \mathbf{I}+\frac{1}{2} n \overline{(\mathbf{v}-\overline{\mathbf{u}}) \cdot \mathbf{I}^{\prime}}=\frac{1}{2} \rho_{1} \epsilon m(\epsilon)|\overline{\mathbf{v}}-\overline{\mathbf{u}}|^{2}+\frac{1}{2} n \overline{\left(\mathbf{v}^{\prime}-\overline{\mathbf{u}}\right) \cdot \mathbf{I}^{\prime}},
$$

where $T$ is again the mean fluid impulse. Due to the averaging process, a term with the mean product of fluctuations appears. One would expect that it is proportional to the square of the mean velocity difference and [37] may therefore be written in the form

$$
\frac{1}{2} n \overline{(\mathbf{v}-\overline{\mathbf{u}}) \cdot \mathbf{I}}=\frac{1}{2} \rho_{1} \in m^{*}|\overline{\mathbf{v}}-\overline{\mathbf{u}}|^{2} .
$$

So, formally, only when the bubbles have equal velocities is it allowed to equate the coefficient $m^{*}(\epsilon)$, appearing in the expression for the mean kinetic energy [38], to the added mass coefficient associated with the fluid impulse.

One final remark should be made: in this paper the bubbly dispersion was considered to be locally uniform, and the configurational pair distribution function was chosen accordingly. The established expression for the fluid impulse therefore does not represent possible effects of small spatial variations ("small deviations from equilibrium"), that in principle should be incorporated in a continuum theory, being essentially a non-equilibrium theory. For flows with variations in the void fraction over length scales that are not "small" in some sense, but "long enough" for a continuum hypothesis to be admissible, the added mass coefficient may also appear to be a function of the local value of the void fraction gradient. There is little doubt, however, that the dependency on the local value of the void fraction itself. given by the above-mentioned relations, remains unaltered in this situation.

Acknowledgements - The authors feel indebted to Professor L. van Wijngaarden for his valuable comments and vigorous criticism during several stimulating discussions. The plan for doing this study was conceived while A.B. was a visitor at Department of Applied Mathematics and Theoretical Physics, University of Cambridge. He wishes to thank Professor H. K. Moffatt (Head of the Department) and Trinity College, Dr E. J. Hinch in particular, for their kind hospitality and The Netherlands Organization for the Advancement of Pure Research (Z.W.O.) for financial support.

\section{REFERENCES}

BatChelor, G. K. 1970 The stress system in a suspension of force-free particles, J. Fluid Mech. $41,545-570$.

BATCHELOR, G. K. 1972 Sedimentation in a dilute suspension of spheres. J. Fluid Mech. 52, 245-268.

Biesheuvel, A. \& GoRissen, W. C. M. 1989 Void fraction disturbances in a uniform bubbly dispersion. Submitted for publication.

Hasimoto, H. 1959 On the periodic fundamental solutions of the Stokes equations and their application to viscous flow past a cubic array of spheres. J. Fluid Mech. 5, 317-328.

Hirschfelder, J. O., CURTISs, C. F. \& BIRD, R. B. 1954 Molecular Theory of Gases and Liquids. Wiley, New York.

JefrRey, D. J. 1973 Conduction through a random suspension of spheres. Proc. R. Soc. Lond. A335, 355-367.

Landau, L. D. \& Lifschitz, E. M. 1959 Fluid Mechanics. Pergamon Press, Oxford.

LighthILL, M. J. 1986 An Informal Introduction to Theoretical Fluid Mechanics. OUP, Oxford.

Milne-Thomson, L. M. 1968 Theoretical Hydrodynamics. Macmillan, London.

SANGani, A. S. \& ACRIvos, A. 1982 Slow flow through a periodic array of spheres. Int. J. Multiphase Flow 8, 343-360.

Sangani, A. S. \& ACRIvos, A. 1983 The effective conductivity of a periodic array of spheres. Proc. R. Soc. Lond. A386, 263-275.

VAN WiJngaARDEN, L. 1976 Hydrodynamic interaction between gas bubbles in liquid. J. Fluid Mech. 77, 27-44.

Zuber, N. 1964 On the dispersed two-phase flow in the laminar flow regime. Chem. Engng Sci. 19, 897-917. 